\title{
Experimental validation of a topology optimized acoustic cavity
}

\author{
Christiansen, Rasmus Ellebæk; Sigmund, Ole; Fernandez Grande, Efren
}

Published in:

Journal of the Acoustical Society of America

Link to article, DOI:

$10.1121 / 1.4936905$

Publication date:

2015

Document Version

Peer reviewed version

Link back to DTU Orbit

Citation (APA):

Christiansen, R. E., Sigmund, O., \& Fernandez Grande, E. (2015). Experimental validation of a topology optimized acoustic cavity. Journal of the Acoustical Society of America, 138(6), 3470-3474.

https://doi.org/10.1121/1.4936905

\section{General rights}

Copyright and moral rights for the publications made accessible in the public portal are retained by the authors and/or other copyright owners and it is a condition of accessing publications that users recognise and abide by the legal requirements associated with these rights.

- Users may download and print one copy of any publication from the public portal for the purpose of private study or research.

- You may not further distribute the material or use it for any profit-making activity or commercial gain

- You may freely distribute the URL identifying the publication in the public portal

If you believe that this document breaches copyright please contact us providing details, and we will remove access to the work immediately and investigate your claim 


\title{
Experimental Validation of a Topology Optimized Acoustic Cavity
}

\author{
Rasmus E. Christiansen ${ }^{\mathrm{a})}$ and Ole Sigmund \\ Section for Solid Mechanics, \\ Department of Mechanical Engineering, \\ Technical University of Denmark (DTU), \\ Building 404, \\ Nils Koppels Allé, \\ DK-2800 Kongens Lyngby, \\ Denmark \\ Efren Fernandez-Grande \\ Acoustic Technology, \\ Department of Electrical Engineering, \\ Technical University of Denmark (DTU), \\ Building 352, \\ Oersteds Plads, \\ DK-2800 Kongens Lyngby, \\ Denmark
}

(Dated: October 27, 2015)

Experimental Validation of a Topology Optimized Acoustic Cavity 1 


\begin{abstract}
This paper presents the experimental validation of an acoustic cavity designed using topology optimization with the goal of minimizing the sound pressure locally for monochromatic excitation. The presented results show good agreement between simulations and measurements. The effect of damping, errors in the production of the cavity and variations in operating frequency is discussed and the importance of taking these factors into account in the modeling process is highlighted.
\end{abstract}

PACS numbers: 43.55.Br, 43.20.Ks, 43.20.Mv, 43.20.Bi 


\section{INTRODUCTION}

Topology optimization ${ }^{2}$ is a method for creating designs optimized for specific objective functions without enforcing a priori restrictions on the design's topology. The method has been used in structural mechanics for more than two decades and has also been successfully applied to other areas of engineering such as fluid mechanics ${ }^{3}$, optics ${ }^{10}$ and $\operatorname{acoustics}^{7,9,12,13}$. Christiansen et $\mathrm{al}^{4}$ and Duhring et $\mathrm{al}^{5}$ have treated the application of topology optimization to the problem of minimizing the sound pressure locally in an acoustic cavity for monochromatic and polychromatic excitation. Despite the interest in applying topology optimization to problems in acoustics, to our knowledge only little work has been reported on the important step of experimentally validating optimized designs for acoustic problems, see e.g. the work by $\mathrm{Akl}$ et $\mathrm{al}^{1}$ and $\mathrm{Lee}^{8}$. In this work we report on the experimental validation of a topology optimized design for an acoustic cavity. This problem was previously addressed by Christiansen et $\mathrm{al}^{4}$, using numerical simulations where the design was optimized to be robust towards small geometric variations that might occur during production or installation. The optimization objective is to provide a localized substantial reduction in pressure in a part of the cavity. Although the considered problem is of a rather academic nature, it is expected that the results regarding experimental validation, calibration of damping properties between experiments and numerical model as well as the study of robustness with respect to manufacturing uncertainties will be important for various real life acoustic design problems within internal automotive noise reduction, hearing aids and other interior acoustic devices. The modeling approach, optimization problem and experimental procedure are outlined, followed by numerical and experimental results.

\footnotetext{
a) Electronic address: raelch@mek.dtu.dk
} 


\section{THE MODEL PROBLEM}

We consider the domain $\Omega \subset \mathbb{R}^{2}$ shown in Figure 1 . The boundary of $\boldsymbol{\Omega}$, denoted $\delta \boldsymbol{\Omega}$, is assumed to be perfectly rigid (fully reflecting) except for the section $\delta \boldsymbol{\Omega}_{\mathbf{P}}$ where a vibrational boundary condition is imposed, modeling a piston vibrating at a single frequency. We seek to minimize the average pressure magnitude, in $\Omega_{\mathrm{OP}}$ by introducing solid material in $\boldsymbol{\Omega}_{\mathrm{d}}$. The experimental measurements of the sound pressure, $p$, were performed in $\boldsymbol{\Omega}_{\mathrm{M}}$.

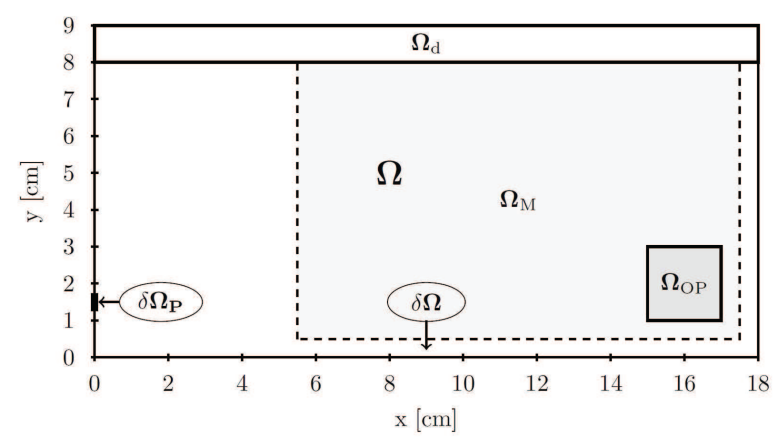

FIG. 1: Model problem diagram. All measures are given in centimeters. Domain:

$\boldsymbol{\Omega}=[0,18] \times[0,9]$. Target sub-domain: $\boldsymbol{\Omega}_{\mathrm{OP}}=[15,17] \times[1,3]$. Design sub-domain: $\boldsymbol{\Omega}_{\mathrm{d}}=[0,18] \times[8,9]$. Experimental measurement sub-domain: $\boldsymbol{\Omega}_{\mathrm{M}}=[5.5,17.5] \times[0.5,7.5]$.

Reflecting boundary: $\delta \boldsymbol{\Omega}$. Excitation boundary: $\delta \boldsymbol{\Omega}_{\mathbf{P}}=0 \times[1.3,1.7]$.

The acoustic field is modeled using the Helmholtz equation along with the appropriate boundary conditions,

$$
\begin{gathered}
\nabla \cdot\left(\rho(\mathbf{x})^{-1} \nabla p(\mathbf{x})\right)+\omega^{2} \kappa(\mathbf{x})^{-1} p(\mathbf{x})=0, \quad \mathbf{x} \in \mathbf{\Omega}, \\
\mathbf{n} \cdot\left(\rho(\mathbf{x})^{-1} \nabla p(\mathbf{x})\right)= \begin{cases}0, & \forall \mathbf{x} \in \delta \boldsymbol{\Omega} \\
-i \omega U, & \forall \mathbf{x} \in \delta \boldsymbol{\Omega}_{\mathbf{P}},\end{cases}
\end{gathered}
$$

where $\mathbf{x}$ denotes the spatial dependence, $\mathrm{i}$ is the imaginary unit, $p$ is the sound pressure, $\omega=2 \pi f$ is the angular frequency, $f$ is the frequency and $U$ is the vibrational velocity of the acoustic source, i.e. the particle velocity at $\delta \boldsymbol{\Omega}_{\mathbf{P}} . \rho(\mathbf{x})$ and $\kappa(\mathbf{x})$ are the density and bulk modulus, respectively, which both depend on the material at point $\mathbf{x}$. The two materials 
considered are solid and air. The contrast in material parameters between solid and air is chosen such that no transverse waves of significant amplitude are excited in solid regions and consequently these waves are excluded from the model. Boundaries of solid regions thereby acts nearly identically to perfectly reflecting boundaries. The values used for the density and bulk modulus of the air are $\rho=1.205 \frac{\mathrm{kg}}{\mathrm{m}^{3}}$ and $\kappa=1.42 \cdot 10^{5} \mathrm{~Pa}$. This choices corresponds to $0 \%$ humidity, a temperature of $20^{\circ} \mathrm{C}$ and a background pressure of $1 \mathrm{~atm}$. The excitation frequency $f=6.942 \mathrm{kHz}$, corresponding to a natural frequency for the empty cavity, is considered. The vibrational velocity $U=0.01 \frac{\mathrm{m}}{\mathrm{s}}$ for the acoustic input is used.

The application of a continuous optimization approach is facilitated by introducing the axillary field, $\xi(\mathbf{x}) \in[0,1] \forall \mathbf{x} \in \boldsymbol{\Omega}_{\mathrm{d}}, \xi(\mathbf{x})=0 \forall \mathbf{x} \in \boldsymbol{\Omega} \backslash \boldsymbol{\Omega}_{\mathrm{d}}$, to interpolate between the inverse material parameters for solid and air as, $\rho(\xi)^{-1}=\rho_{\text {air }}^{-1}+\xi\left(\rho_{\text {solid }}^{-1}-\rho_{\text {air }}^{-1}\right)$ and $\kappa(\xi)^{-1}=$ $\kappa_{\text {air }}^{-1}+\xi\left(\kappa_{\text {solid }}^{-1}-\kappa_{\text {air }}^{-1}\right)$. This material interpolation is chosen based on the work by ${ }^{5}$. It is the natural interpolation since the inverse material parameters appear directly in the Helmholtz equation. The minimization of the average pressure level in $\boldsymbol{\Omega}_{\mathrm{OP}}$ may be stated as the continuous optimization problem,

$$
\begin{aligned}
& \min _{\xi} . \Phi(\xi)=\frac{1}{\int d \boldsymbol{\Omega}_{\mathrm{OP}}} \int|p(\mathbf{x}, \xi)|^{2} d \boldsymbol{\Omega}_{\mathrm{OP}}, \\
& \text { s.t. } 0 \leq \xi(\mathbf{x}) \leq 1 \quad \forall \mathbf{x} \in \boldsymbol{\Omega}_{\mathrm{d}},
\end{aligned}
$$

where $p(\mathbf{x}, \xi)$ is obtained by solving (1) for a given realization of $\xi$. We used topology optimization in conjunction with adjoint sensitivity analysis and the gradient based optimization method, The Method of Moving Asymptotes ${ }^{11}$, to solve (2). For the optimization procedure we consider a modified version of (2) using filtering, including smoothing and projection with a continuation scheme on the projection strength. This approach results in near-perfect solid/void designs as a direct result of the optimization, thus limiting the need for post processing. In addition the design has been optimized for both uniform and non-uniform perturbations of the geometry to assure high performance if any errors are introduced in the geometry during production, installation or use. The stopping criteria 
used for in optimization process is $\left|\Phi_{n}-\max _{j \in n-10, . ., n-1}\left(\Phi_{j}\right)\right|<0.01 \Phi_{n}$, where $\Phi_{n}$ is objective value at the n'th iteration. The interested reader is referred to Christiansen $\mathrm{et}^{\mathrm{al}}{ }^{4}$ for further details on the modeling and optimization process. To obtain the final design used as a blueprint for production a threshold at $\xi(\mathbf{x})=0.5$ is applied to the optimized design. For the design treated in the following below $0.2 \%$ of the optimized density distribution was composed of values different from either fully solid or fully air.

\section{NUMERICAL RESULTS}

The final design obtained from the optimization process, along with contours indicating the bounds of the uniform and non-uniform geometric perturbations for which the design was optimized, are shown in Figure 2.

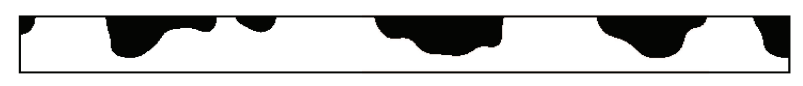

(a)

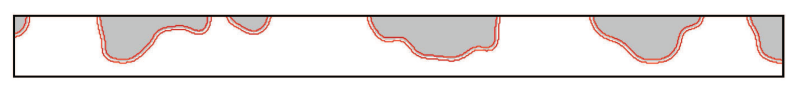

(b)

FIG. 2: (color online) Design optimized for local pressure minimization in $\boldsymbol{\Omega}_{\mathrm{OP}}$ at $f=6.942 \mathrm{kHz}$ (corresponding to $\Omega_{\mathrm{d}}$ in the acoustic cavity shown in Figure 1 ). (a) Final design, Black is solid and white is air. (b) Bounds on geometric perturbations considered in the optimization process. The final design is shown in gray and the bounds on the geometric perturbations considered in the optimization are outlined.

Figure 3a shows a plot of the simulated pressure field, reported in dB SPL ref $20 \mu \mathrm{Pa}$, for the model problem given in Figure 1 with the design presented in Figure 2 introduced in $\Omega_{\mathrm{d}}$. The pressure is seen to be significantly lowered in $\boldsymbol{\Omega}_{\mathrm{OP}}$ compared to the rest of $\boldsymbol{\Omega}$. Figure $3 \mathrm{~b}$ shows the simulated pressure in the empty cavity. The average sound pressure in $\Omega_{\mathrm{OP}}$ in the empty cavity is, $\langle L\rangle_{\boldsymbol{\Omega}_{\mathrm{OP}, \text { no design }}}=125 \mathrm{~dB}$, while this average is reduced to 
$\langle L\rangle_{\boldsymbol{\Omega}_{\mathrm{OP}, \mathrm{design}}}=44.9 \mathrm{~dB}$, when the design is introduced in $\boldsymbol{\Omega}_{\mathrm{d}}$.

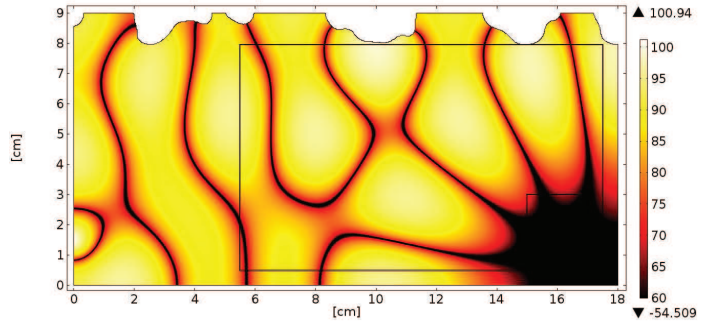

(a)

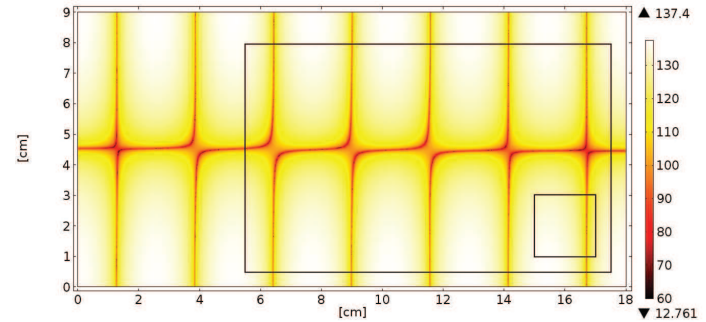

(b)

FIG. 3: (color online) Simulated pressure [dB SPL ref $20 \mu \mathrm{Pa}]$ in the cavity at $f=6.942$ kHz. $\Omega_{\mathrm{M}}$ and $\Omega_{\mathrm{OP}}$ are outlined using thin black lines. (a) Optimized design introduced.

(b) Empty cavity. Note the difference in the maximal dB value between (a) and (b).

An extruded 3D model of the cavity containing the optimized design is created using COMSOL version $4.3 \mathrm{~b}$. The extrusion distance is chosen to be $0.6 \mathrm{~cm}$ which is large enough for experimental practicalities while remaining much smaller than the wavelength of the monochromatic excitation to assure that the pressure field remains two dimensional inside the cavity (the cutoff frequency in the extruded dimension is around $30 \mathrm{kHz}$. Below the cutoff frequency the sound field in the cavity remains two dimensional). A full elasto-acoustic multi-physics model is applied to confirm the pressure field in the extruded cavity. The model accounts for acoustic waves in air regions and transverse elastic waves in solid regions. The material parameters of the solid are set to $\rho=9.54 \cdot 10^{2} \frac{\mathrm{kg}}{\mathrm{m}^{3}}$ and $\kappa=1.9 \mathrm{GPa}$ respectively, corresponding to measured values for the ABS plastic used for the fabrication. The investigation confirms that no transverse waves of significant amplitude are excited in the solid regions and that the pressure field remains two dimensional inside the cavity.

\section{EXPERIMENTAL RESULTS}

A solid open faced version of the extruded design and cavity, shown in Figure 4, is produced in ABS plastic using 3D-printing. The open faced cavity is produced in two parts to allow for 
testing multiple designs with ease. The top part contains the design while the bottom part consists of the remaining part of the cavity. The bottom part has a circular hole of $4 \mathrm{~mm}$ diameter on the side corresponding to the section $\delta \boldsymbol{\Omega}_{\mathbf{P}}$ shown in Figure 1. The dimensions of the printed design are given in table I.

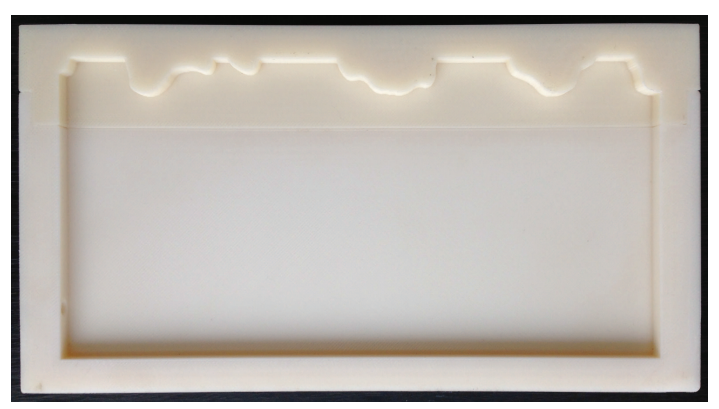

FIG. 4: (color online) Extruded 3D-printed design and cavity.

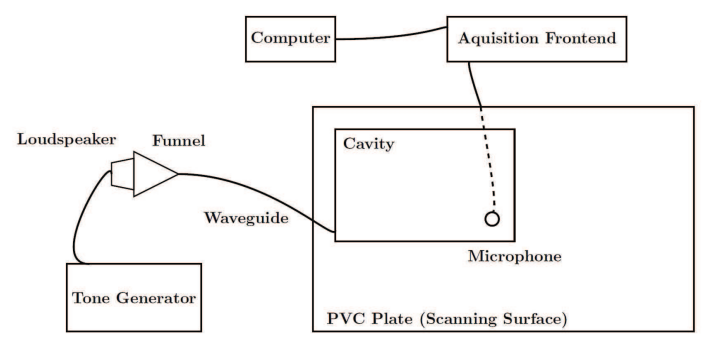

FIG. 5: Schematics illustrating experimental setup. The cavity and PVC plate can slide relative to each other to scan the microphone across the cavity.

The experimental setup, see Figure 5, consists of the 3D-printed open face cavity placed face down on a $1 \mathrm{~cm}$ thick PVC plate with a $1 / 8$ inch B\&K microphone flush mounted in its center. The cavity is movable, making it possible to scan the sound field inside it. The source consists of a 3 inch loudspeaker connected to a waveguide that radiates into the cavity through the hole at $\delta \boldsymbol{\Omega}_{\mathbf{P}}$. The temperature and humidity is measured and the excitation frequency adjusted to account for perturbations of $\rho$ and $\kappa$, compared to the values used in the simulation. This adjustment results in a frequency of, $f_{\text {adj }}=7.011$ $\mathrm{kHz}$ (monochromatic excitation). The microphone is connected to a B\&K Pulse analyzer. 
The pressure field is measured in a regular grid, in $\Omega_{\mathrm{M}}$, with the inter-spacing between each measurement point being $5 \mathrm{~mm}$ in both spatial directions. The positioning of the scanning system is adjusted manually to within $0.5 \mathrm{~mm}$ accuracy for each measurement. The measurements are performed with a spectral resolution of $1 \mathrm{~Hz}$ centered at $f_{\text {adj }}$. The background noise level in the cavity was measured at $55 \mathrm{~dB}$ SPL providing a lower limit on the measurements and a way of estimating the signal to noise ratio.

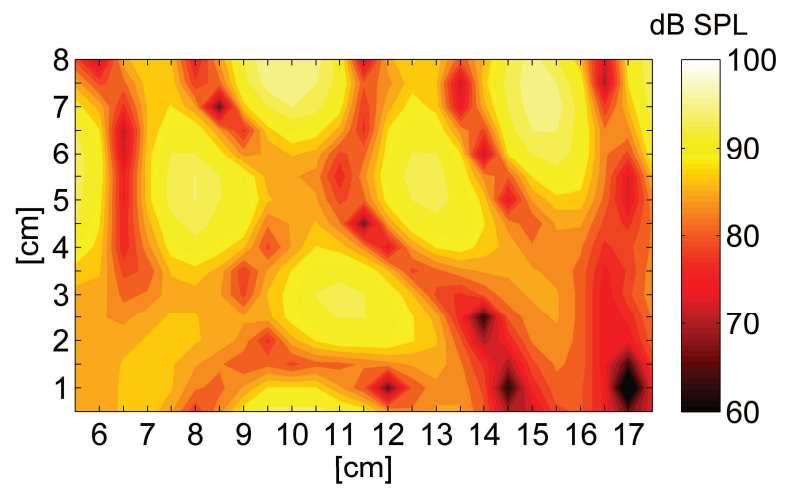

(a)

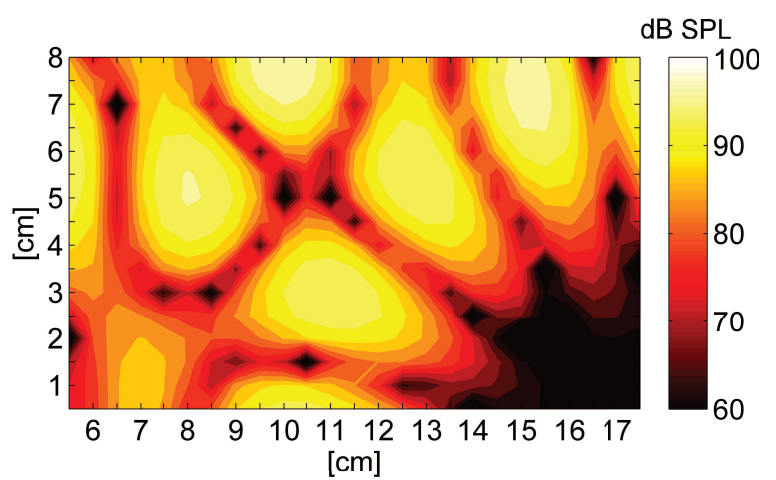

(b)

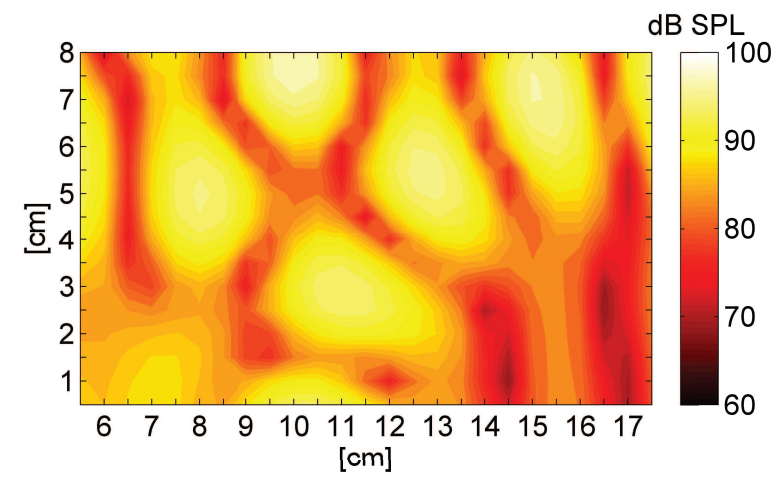

(c)

FIG. 6: (color online) Contour plot of sound pressure field in $\Omega_{\mathrm{M}}$, (a) Experimentally measured field for the 3D-printed acoustic cavity. (b) Simulated field. (c) Simulated field with mass proportional damping. (b) and (c) The field is smoothed with a constant filter with $1 / 16$ inch $(\approx 0.159 \mathrm{~cm})$ radius, sampled at the measurement positions.

Figure 6a presents a contour plot of the measured sound pressure field in $\Omega_{\mathrm{M}}$. For compari- 
son Figure $6 \mathrm{~b}$ shows a contour plot of the simulated pressure field in $\Omega_{\mathrm{M}}$, smoothed using a constant filter, with a radius of $1 / 16$ inch $(\approx 0.159 \mathrm{~cm})$, to mimic the resolution of the microphone, sampled at the positions where the experimental measurements were performed. The result presented in Figure $6 \mathrm{~b}$ is obtained from a simulation where the modeling domain dimensions are chosen to be identical to those of the 3D-printed open faced cavity to a precision of $0.02 \mathrm{~cm}$, (corresponding to the accuracy of the 3D-printer used for production). This change in the numerical model results in changes in the simulated pressure field compared to Figure 3a mainly observable in low pressure regions. By comparing the measured result with the simulated result good qualitative agreement is found. The position of the nodal lines and pressure maxima agree with only some discrepancy in the lower left and right corners of $\Omega_{\mathrm{M}}$. A difference in the pressure magnitude close to nodal lines is observed. This difference can be explained partly by the background noise in the measurements, both due to transducer noise, and due to background noise in the measurements caused by sound being transmitted through the walls of the acoustic cavity. Also, close to the nodal lines, the sound field changes rapidly. Hence small positioning errors of the microphone result in significant deviations in the measured pressure. Finally the finite size of the microphone results in a spatial averaging, particularly notable around the nodal lines. As mentioned above, near $\Omega_{\mathrm{OP}}$ and in the lower left corner of $\Omega_{\mathrm{M}}$ there are noticeable discrepancies in the pressure field, with the measurement result showing a higher sound pressure. A reasonable explanation for the difference between the measured and simulated pressure field is the existence of damping in the physical cavity which was neglected in the simulation and optimization. In short, damping inside the rigid cavity occurs mostly because the process tends to be isothermal close to rigid walls, rather than adiabatic as is assumed in the derivation of the Helmholtz equation. In addition there are also viscous losses in the system. A description of damping for room acoustics may be found in chapter 8 of the book by Jacobsen and $\mathrm{Juhl}^{6}$. To test this theory mass proportional damping is introduced in the computer model by adding the term $i \eta \omega p(\mathbf{x})$, to equation (1) where $\eta$ is a freely chosen parameter. A parameter study for $\eta$ is performed to find the amount of damping which 
provides the smallest discrepancies between the measurements and the simulation in $\Omega_{\mathrm{M}}$. From this study $\eta=0.02$ is obtained. Figure $6 \mathrm{c}$ shows a simulation result obtained using the mass proportional damping with $\eta=0.02$. In this simulation the vibrational velocity is increased to $U=0.013 \frac{\mathrm{m}}{\mathrm{s}}$ to account for the overall decrease in the sound pressure caused by the damping. Comparing the pressure field in Figure $6 \mathrm{c}$ to the pressure fields in Figure 6a and Figure 6b, better overall agreement between simulation and measurement are found when damping is included. It is seen that the discrepancies between simulation and measurement in the area around $\Omega_{\mathrm{OP}}$ and in the bottom left part of $\boldsymbol{\Omega}_{\mathrm{M}}$ have decreased significantly. In addition, a better agreement for the pressure along the nodal lines has been obtained. At the same time no significant deterioration in the agreement of the two results elsewhere in $\Omega_{\mathrm{M}}$ is observed. This finding supports that the inclusion of mass proportional damping results in more realistic modeling for the investigated case of an extruded 2D cavity.

In addition to the possible effect of damping, the discrepancy between the undamped simulation result and the measurements may be caused by the following. The model problem is sensitive to geometric perturbations of the cavity elsewhere than in $\boldsymbol{\Omega}_{\mathrm{d}}$, as seen from the difference in $\langle L\rangle_{\boldsymbol{\Omega}_{\mathrm{OP}, \text { sim }}}$ between the result presented in Figure $6 \mathrm{~b}$ and the result presented in Figure 3a. The problem is also sensitive towards the source position and the operating frequency. The sensitivity towards the operating frequency was observed from measurements performed without the adjustment of the operating frequency from $f=6.942$ $\mathrm{kHz}$ to $f_{\text {adj }}=7.011 \mathrm{kHz}$ where the performance of the design decreased in terms of the value of $\langle L\rangle_{\boldsymbol{\Omega}_{\mathrm{OP}, \text { sim }}}$ (not shown). This sensitivity is due to the main effect responsible for producing a localized low sound pressure being the alignment of nodal lines in $\boldsymbol{\Omega}_{\mathrm{OP}}$. Such an alignment is highly sensitive to small changes in geometry, frequency and source position.

The average sound pressure in $\Omega_{\mathrm{OP}}$ for the experimental measurement is, $\langle L\rangle_{\boldsymbol{\Omega}_{\mathrm{OP}, \text { mea }}} \approx 78.6$ $\mathrm{dB}$. From the simulation result reported in Figure $6 \mathrm{~b},\{L\}_{\boldsymbol{\Omega}_{\mathrm{OP}, \text { sim }}}=59.1 \mathrm{~dB}$, is obtained, while for the simulation including the damping it is $\langle L\rangle_{\Omega_{\mathrm{OP}, \text { sim }, \mathrm{damp}}} \approx 78.1 \mathrm{~dB}$. The difference 
between $\langle L\rangle_{\boldsymbol{\Omega}_{\mathrm{OP}, \text { sim }}}$ and $\langle L\rangle_{\boldsymbol{\Omega}_{\mathrm{OP}, \mathrm{mea}}}$ is approximately $19.5 \mathrm{~dB}$ whereas this difference between $\langle L\rangle_{\boldsymbol{\Omega}_{\mathrm{OP}, \text { sim,damp }}}$ and $\langle L\rangle_{\boldsymbol{\Omega}_{\mathrm{OP}, \text { mea }}}$ is $0.5 \mathrm{~dB}$. In spite of the difference between $\langle L\rangle_{\boldsymbol{\Omega}_{\mathrm{OP}, \text { sim }}}$ and $\langle L\rangle_{\boldsymbol{\Omega}_{\mathrm{OP}, \mathrm{mea}}}$ the experimentally verified reduction in sound pressure in $\boldsymbol{\Omega}_{\mathrm{OP}}$ is still significant compared to the sound pressure elsewhere in the cavity and to the sound pressure in the empty cavity, see Figure 3b.

As general lessons to be learned with regards to using topology optimization for acoustic design problems we list the following. To minimize the discrepancy between the predicted and experimental performance of the design it is suggested to take manufacturing uncertainties and uncertainties in other model parameters into account in the modeling process. For example accounting for small changes in the operating frequency which may be caused by changes in humidity and/or temperature. It is also suggested to include an amount of damping in the model corresponding to experimental findings. Using these suggestions may allow the final design to maintain the predicted performance under a range of varying real world operating conditions.

The focus of the present study was on experimentally validating a design, created using topology optimization, optimized to perform well under a range of geometric perturbations, under monochromatic excitation. It is straight forward to extend the topology optimization method applied in this paper to wider frequency bands. As the frequency band is widened the sensitivity of the average response in $\Omega_{\mathrm{OP}}$ to geometric variations and the possible reduction in sound pressure are both expected to be reduced, c.f. ${ }^{4}$.

\section{CONCLUSION}

An approach for experimentally measuring the pressure field in a 2D acoustic cavity was presented. An optimized design created using topology optimization was tested and good agreement between the simulated and the measured pressure field was shown. The presented results illustrate the usefulness of the approach as a tool for designing acoustic cavities for 
local pressure minimization. A description of the steps needed to assure that the physical design functions as predicted by the pure acoustic model was outlined. It was furthermore shown that adding mass proportional damping to the mathematical model improves the agreement between simulation and measurement, suggesting the inclusion of damping in the design process will result in better agreement between predicted and measured results. Finally it was highlighted that the inclusion of manufacturing uncertainties and operating conditions in the design process, is important to minimize the discrepancies between the mathematical model and the final physical design when considering sensitive problems.

\section{ACKNOWLEDGMENTS}

The 3D-printing laboratory FabLab at the Technical University of Denmark produced the optimized design and acoustic cavity. Assistant Engineer Jørgen Rasmussen at the institute for electrical engineering at the Technical University of Denmark helped with preparing the experiment. Niklas Flink Hansen and Peter Toftemann performed the measurements of the material parameters for the ABS plastic used in the 3D-printing process. The work was financially supported by Villum Fonden through the research project Topology Optimization - the Next Generation (NextOpt).

\section{References}

1 W. Akl, A. El-Sabbagh, K. Al-Mitani, A. Baz, Topology optimization of a plate coupled with acoustic cavity. International Journal of Solids and Structures, Elsevier, 46, pp. 2060-2074 (2009)

2 M. P. Bendsøe, O. Sigmund, Topology Optimization. Springer-Verlag Berlin Heidelberg, 2nd edition, pp. 370 (2003)

3 T. Borrvall, J. Petersson, Topology optimization of fluid in stokes flow. International Journal for Numerical Methods in Fluids, Wiley, 41 pp. 77-107 (2003). 
4 R. E. Christiansen, B. S. Lazarov, J. S. Jensen, O. Sigmund, Creating geometrically robust designs for highly sensitive problems using topology optimization - acoustic cavity design. Structural and Multidiciplinary Optimization, Springer, [DOI 10.1007/s00158-015-12655] (2015).

5 M. B. Dühring, J. S. Jensen, O. Sigmund, Acoustic design by topology optimization. Journal of Sound and Vibration, Elsevier, 317, pp. 557-575 (2008).

${ }^{6}$ F. Jacobsen, P. M. Juhl, Fundamentals of General Linear Acoustics. John Wiley \& Sons Ltd, The Atrium, Southern Gate, Chichester, West Sussex, PO19 8SQ, United Kingdom, pp. $127-157$ (2013).

7 J. Kook, K. Koo, J. Hyun, J. S. Jensen, S. Wang, Acoustical topology optimization for zwicker's loudness model - application to noise barriers. Computational Methods for Applied Mechanical Engineering, Elsevier, 237, pp. 130-151 (2012).

8 J. W. Lee, Optimal topology of reactive muffler achieving target transmission loss values: Design and experiment. Applied Acoustics, Elsevier, 88, pp. 104-113 (2015).

9 J. W. Lee, Y. Y. Kim, Rigid body modeling issue in acoustic topology optimization. Computational Methods for Applied Mechanical Engineering, Elsevier, 198, pp. 1017-1030 (2009).

10 O. Sigmund, J. S. Jensen, Topology optimization for nano-photonics. Laser \& Photonics Reviews, Wiley, 5, pp. 308-321 (2011).

11 K. Svanberg, The method of moving asymptotes - a new method for structural optimization. International Journal for Numerical Methods in Engineering, Wiley, 24, pp. 359-373 (1987).

12 E. Wadbro, Analysis and design of acoustic transition sections for impedance matching and mode conversion., Structural and Multidiciplinary Optimization, Springer, 50, pp. 395-408 (2014).

13 E. Wadbro, M. Berggren, Topology optimization of an acoustic horn. Computational Methods for Applied Mechanical Engineering, Elsevier, 196, pp. 420-436 (2006). 
TABLE I: Cavity and PVC plate data. The inner dimensions of the cavity without the design are reported to within $0.02 \mathrm{~cm}$ accuracy.

\begin{tabular}{l|c} 
Component & $(\mathrm{x}, \mathrm{y}, \mathrm{z})$ \\
\hline Cavity (Top), $[\mathrm{cm}]$ & $18.00 \times 2.00 \times 0.60$ \\
Cavity (Bottom), $[\mathrm{cm}]$ & $18.02 \times 7.00 \times 0.60$ \\
Wall Thickness $[\mathrm{cm}]$ & $1.2 \times 1.2 \times 1.0$ \\
PVC plate $[\mathrm{cm}]$ & $40.0 \times 20.0 \times 1.0$ \\
\hline
\end{tabular}




\section{List of Figures}

FIG. 1 Model problem diagram. All measures are given in centimeters. Domain: $\boldsymbol{\Omega}=[0,18] \times[0,9]$. Target sub-domain: $\boldsymbol{\Omega}_{\mathrm{OP}}=[15,17] \times[1,3]$. Design sub-domain: $\boldsymbol{\Omega}_{\mathrm{d}}=[0,18] \times[8,9]$. Experimental measurement sub-domain: $\Omega_{\mathrm{M}}=[5.5,17.5] \times[0.5,7.5]$. Reflecting boundary: $\delta \Omega$. Excitation boundary:

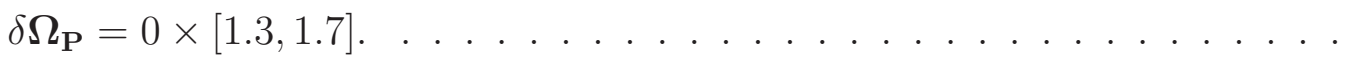

FIG. 2 (color online) Design optimized for local pressure minimization in $\boldsymbol{\Omega}_{\mathrm{OP}}$ at $f=6.942 \mathrm{kHz}$ (corresponding to $\boldsymbol{\Omega}_{\mathrm{d}}$ in the acoustic cavity shown in Figure ??). (a) Final design, Black is solid and white is air. (b) Bounds on geometric perturbations considered in the optimization process. The final design is shown in gray and the bounds on the geometric perturbations considered in the optimization are outlined.

FIG. 3 (color online) Simulated pressure [dB SPL ref $20 \mu \mathrm{Pa}]$ in the cavity at $f=$ $6.942 \mathrm{kHz} . \Omega_{\mathrm{M}}$ and $\Omega_{\mathrm{OP}}$ are outlined using thin black lines. (a) Optimized design introduced. (b) Empty cavity. Note the difference in the maximal dB value between $(\mathrm{a})$ and $(\mathrm{b}) \ldots \ldots \ldots \ldots \ldots$

FIG. 4 (color online) Extruded 3D-printed design and cavity. . . . . . . . . . 8

FIG. 5 Schematics illustrating experimental setup. The cavity and PVC plate can slide relative to each other to scan the microphone across the cavity. . . .

FIG. 6 (color online) Contour plot of sound pressure field in $\Omega_{\mathrm{M}}$, (a) Experimentally measured field for the 3D-printed acoustic cavity. (b) Simulated field. (c) Simulated field with mass proportional damping. (b) and (c) The field is smoothed with a constant filter with $1 / 16$ inch $(\approx 0.159 \mathrm{~cm})$ radius, sampled at the measurement positions. 